\title{
Single Piezoelectric Transducer as Strain Sensor and Energy Harvester Using Time- multiplexing Operation
}

\author{
Zheng Jun Chew, Member, Tingwen Ruan, Meiling Zhu, Member, Marise Bafleur, Senior \\ Member, and Jean-Marie Dilhac
}

\begin{abstract}
This paper presents the implementation of a single piece of macro-fiber composite (MFC) piezoelectric transducer as a multifunctional device for both strain sensing and energy harvesting for the first time in the context of an energy harvesting powered wireless sensing system. The multifunction device is achieved via timemultiplexing operation for alternating dynamic strain sensing and energy harvesting functions at different time slots associated with different energy levels, that is, when there is insufficient energy harvested in the energy storage for powering the system, the MFC is used as an energy harvester for charging up the storage capacitor; otherwise, the harvested energy is used for powering the system and the MFC is used as a strain sensor for measuring dynamic structural strain. A circuit is designed and implemented to manage the single piece of MFC as the multifunctional device in a time-multiplexing manner, and the operation is validated by the experimental results. The dynamic strains measured by the MFC in the implemented system match a commercial strain sensor of extensometer by 95.5 to $99.99 \%$, and thus the studied method can be used for autonomous structural health monitoring of dynamic strain.
\end{abstract}

Index Terms-energy harvesting, macro fiber composite, multifunctional device, strain sensor, timemultiplexing operation.

\section{INTRODUCTION}

$\mathrm{S}_{\mathrm{a}}^{\mathrm{T}}$ TRAIN is one of the most widely measured quantities to assess whether the loading and fatigue of engineering structure is within safe load levels or not in structural health monitoring applications. Traditionally, strain is often measured using metal foiled wire strain gauges [1]. This measurement method is simple and reliable but requires wire installation associated with circuits interfacing the strain gauges that are bulky and power hungry, thus limiting its wide deployment in real engineering structures for active services.

The widespread of wireless sensor networks (WSNs) sees a paradigm shift in many wired sensor systems including strain

This work was supported in part by the Engineering and Physical Sciences Research Council, U.K., through the Projects SMARTERSmart Multifunction Architecture and Technology for Energy-Aware Wireless Sensors under Grant EP/K017950/1. All data are provided in full in the results section of this paper. measurement systems because WSNs are easier and cheaper to be deployed [2]. However, most WSNs are powered by batteries which have limited lifespan and need to be replaced regularly. Battery-free WSNs can be realized by harvesting ambient energy as their energy source through energy harvesting to power up the system [3]. Vibration energy is one of the most attractive sources due to its abundance in engineering structures [4], [5]. Piezoelectric transducers are widely used as vibration energy harvesters due to their simple structures and high power generation capability [6], [7]. Strain induced on a structure due to vibration can be harvested using flexible patch type piezoelectric transducers such as macro fiber composite (MFC), which is directly bonded onto the host structure to convert the dynamic strain experienced by the host structure into electrical energy [5], [6].

Piezoelectric transducers can response to very low frequencies, for example, $0.1 \mathrm{~Hz}$ [8]. Therefore, apart from strain energy harvesting, piezoelectric transducer can also be used for dynamic strain sensing, where MFC attached to a host structure in a similar way to energy harvesting so that the dynamic strain experienced by the host structure can be directly measured by the MFC strain sensor has been reported in [9], [10]. The voltage generated by the MFC in an opencircuit can be taken as the sensor reading. In addition, the MFC is highly flexible, which allows it to be bonded onto a host structure with non-flat surfaces for strain measurement [9], [10], and has no moving parts, making it more robust and reliable in severe environment such as the aeronautics one. Furthermore, as a strain sensor itself, the MFC does not require any external power supply for operation [10]. In spite of the significant advantages of MFC as energy harvesters and strain sensors, no work has been reported on using a single piece of MFC as a multifunctional device for both strain sensing and energy harvesting so far. Most of the reported work is implemented using two independent piezoelectric transducers [11], [12]. It should be noted that the transducer which is used as sensor will only be functioning when enough energy has been harvested from the other transducer which is used as a harvester.

This paper herein presents the implementation of a single piece of MFC piezoelectric transducer as a multifunctional device for strain sensing and energy harvesting for the first time in the context of energy-autonomous wireless sensing 


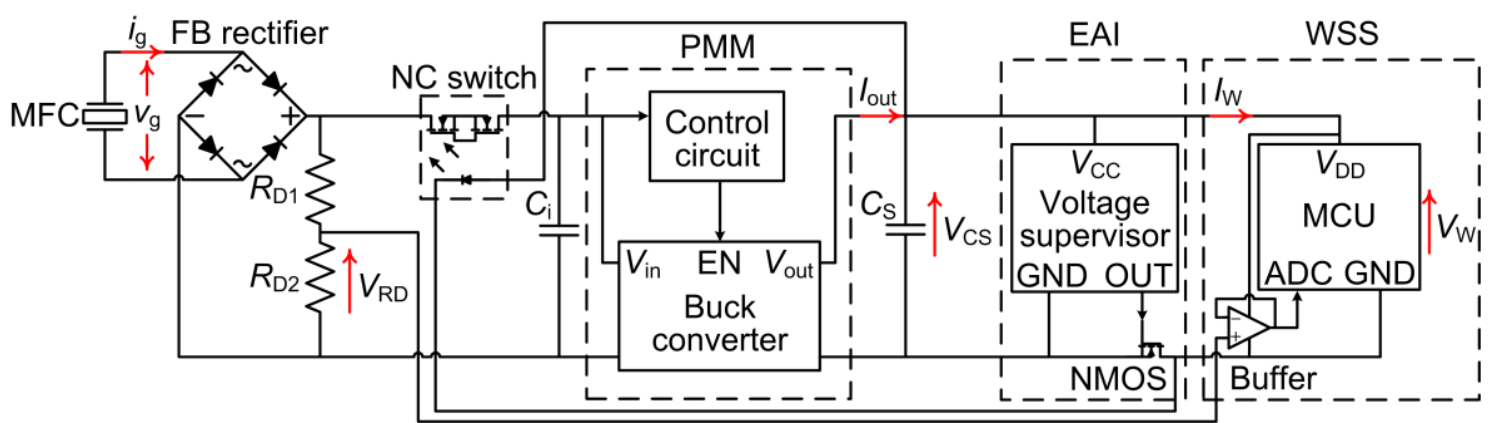

Fig. 1. Schematic of the implemented system using a single piece of macro-fiber composite (MFC) piezoelectric transducer as a multifunctional device for strain sensing and energy harvesting in energy-autonomous wireless sensing system.

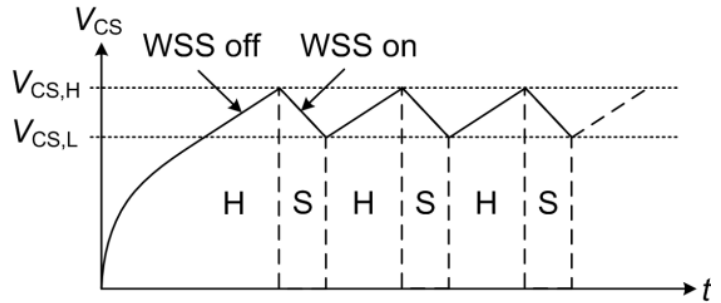

Fig. 2. The time-multiplexing operation of the implemented system, where " $\mathrm{H}$ " represents the time slot that the same piece of MFC is used for energy harvesting and " $\mathrm{S}$ " for dynamic strain sensing.

system (WSS). This innovation eliminates the need of two individual piezoelectric transducers for energy harvesting and sensing purposes, respectively, reducing size and cost. The MFC is used as the multifunctional device in a timemultiplexing manner where the MFC is used alternately as either a strain energy harvester or a strain sensor based on the energy availability to the system. A circuit is designed and implemented to manage the single piece of MFC as a multifunctional sensing and harvesting device in a timemultiplexing manner and validated by experimental results.

\section{SYSTEM DESCRIPTION AND OPERATION}

Fig. 1 shows the schematic of the implemented energy harvesting powered dynamic strain sensing system using a single piece of MFC piezoelectric transducer as a multifunctional device for strain sensing and energy harvesting. The system is composed of a full wave diode bridge (FB) rectifier, a power management module (PMM), a storage capacitor $\left(C_{\mathrm{S}}\right)$, an energy-aware interface $(\mathrm{EAI})$, and a wireless sensing system (WSS), which is similar to the previously reported system architectures [13], [14] but with the following additional features to manage the single piece of MFC as a multifunctional device:

1) Time-multiplexing operation realized through a normally closed (NC) switch that alternates dynamic strain sensing and energy harvesting functions of the MFC at different time slots based on the energy level to the system. The $\mathrm{NC}$ switch is controlled by the voltage supervisor in the energy-aware interface.

2) Voltage $V_{\mathrm{RD}}$ as the sensor reading with a buffer to minimize loading effect on the MFC which arises from the WSS, where $V_{\mathrm{RD}}$ is taken from a resistive network formed by resistors $R_{\mathrm{D} 1}$ and $R_{\mathrm{D} 2}$.

\section{A. Time-multiplexing Operation}

Fig. 2 shows the time-multiplexing operation using a single piece of MFC piezoelectric transducer as both sensor and energy harvester at different time slots associated with different energy levels, represented by the voltage of the storage capacitor that has a high threshold voltage $V_{\mathrm{CS}, \mathrm{H}}$ and a low threshold voltage $V_{\mathrm{CS}, \mathrm{L}}$. When there is insufficient energy in the storage capacitor $C_{\mathrm{S}}$ to power the system, the WSS is off and the MFC is used as an energy harvester, represented by " $\mathrm{H}$ " in Fig. 2, to charge up the storage capacitor until the voltage reaches $V_{\mathrm{CS}, \mathrm{H}}$; otherwise, the harvested energy which is accumulated in $C_{\mathrm{S}}$ is used to power the developed WSS, and the MFC is used as a strain sensor, represented by "S" in Fig. 2 , to measure the strain experienced by the host structure until the voltage drops to $V_{\mathrm{CS}, \mathrm{L}}$. This time-multiplexing operation is repeated as long as the MFC is subjected to strain loading induced by the vibration on the host structure.

\section{B. System Implementation for Time-multiplexing Operation}

The NC switch is for making or breaking the connection between the FB rectifier and the PMM so that the MFC can be used as either a strain energy harvester or a strain sensor. The $\mathrm{NC}$ switch is closed when the system is in energy harvesting mode, allowing the energy generated by the MFC to flow through the $\mathrm{NC}$ switch to be stored in the storage capacitor by the PMM. The NC switch can be of any type but a solid-state relay (LH1511) is chosen here because it can withstand high voltage and provide good isolation when it breaks the connection. The solid-state relay is closed when no electrical energy is supplied to it. It becomes opened when electrical energy is supplied to its infrared LED, thus isolating the MFC from the PMM and enabling the MFC to serve as a strain sensor. This means the solid-state relay consumes energy only when it is opened for a short period of time.

Rectified DC electrical energy from the FB rectifier built of four 1N4148 diodes that flows through the NC switch will be smooth out by the smoothing capacitor $C_{\mathrm{i}}$. The PMM is mainly used for managing energy transfer from the MFC to the storage capacitor by converting the smoothened DC voltage to a voltage of no more than $3.3 \mathrm{~V}$ to charge up the storage capacitor for use by the WSS. It should be mentioned that the PMM can be of any type as long as it can convert the voltage from the MFC to a suitable level for the WSS. In this 


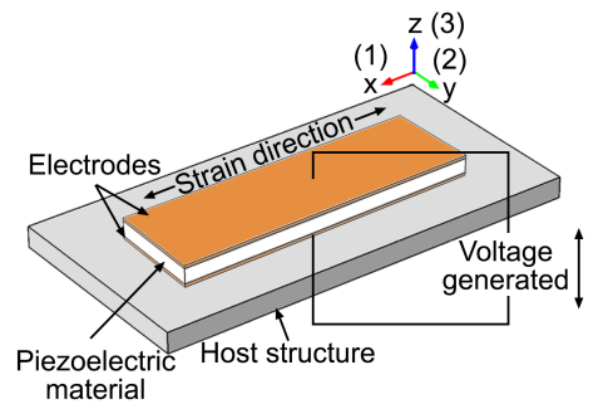

(a)

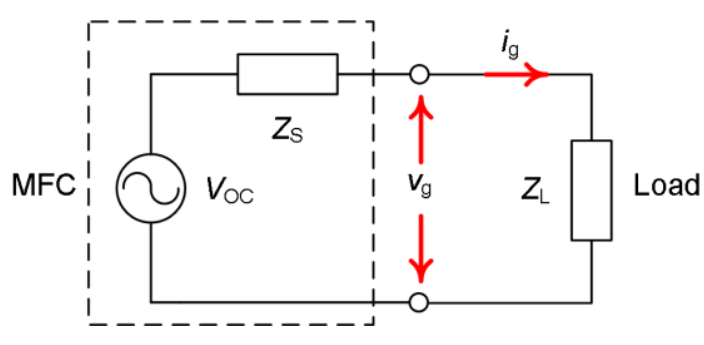

(b)

Fig. 3. Illustration of (a) a piezoelectric transducer with one side bonded on a host structure that operates in the 31 mode and (b) an equivalent circuit model of an MFC represented by the circuit in the dash lines which is connected to an external load as energy harvester.

system, the custom-developed PMM has a maximum power point finding control circuit which operates based on the wellproven half-open-circuit voltage method $\left(V_{\mathrm{OC}} / 2\right)$ [7], [15]. The buck converter will only be enabled momentarily for maximum power transfer of the MFC at the voltage with the amplitude of $\left|V_{\mathrm{OC}}\right| / 2$. Details of the PMM will not be discussed here since the focus of this paper is on the implementation of the time-multiplexing method for the multifunctional device.

The size of storage capacitor influences the turn on and turn off time of the WSS, where a larger capacitor takes a longer time to be charged up but also keeps the WSS on for a longer time and vice versa. Given that the average current consumption of a ZigBee wireless sensor node is approximately $7 \mathrm{~mA}$ [16], a $22 \mathrm{mF}$ capacitor is chosen where the value can keep the WSS on for a time duration of about 2 seconds for strain measurement.

The EAI is composed of a voltage supervisor (LTC2935-1) with a set of fixed voltage thresholds and an NMOS to control the energy flow from the storage capacitor to the WSS. The voltage supervisor monitors the voltage across the storage capacitor and turns the NMOS on when the voltage is at a high threshold voltage $V_{\mathrm{CS}, \mathrm{H}}$, for sensing function, or off at a low threshold voltage $V_{\mathrm{CS}, \mathrm{L}}$, for energy harvesting function of the implemented system, as shown in Fig. 2. When the NMOS is turned on, the ground (GND) pin of the WSS is connected to the system ground and forms a closed circuit with the storage capacitor, enabling energy to be drawn out from the storage capacitor which subsequently turns the WSS on. The capacitor voltage $V_{\mathrm{CS}}$ decreases as energy is being drawn. The WSS will be completely turned off with zero sleeping current when the NMOS is switched off by the voltage supervisor as $V_{\mathrm{CS}}$ reaches $V_{\mathrm{CS}, \mathrm{L}}$ to allow energy to be accumulated. Although the voltage supervisor is consuming some energy during the energy accumulation, it is negligible since the current consumption is less than $1 \mu \mathrm{A}$ [17]. The EAI also acts as a voltage regulator by maintaining the voltage supplied to the WSS between $V_{\mathrm{CS}, \mathrm{L}}$ and $V_{\mathrm{CS}, \mathrm{H}}$.

The EAI is also used to toggle the NC switch which sits in between the FB rectifier and the PMM in a similar way as controlling the WSS by cutting off or providing energy to the infrared LED of the NC switch. The MFC is used as a strain energy harvester when the $\mathrm{NC}$ switch is closed, connecting the MFC together with the FB rectifier to the PMM. The MFC is used as a strain sensor when the NC switch is opened, where the FB rectifier together with the MFC is disconnected from the PMM. Therefore, the EAI serves as the main control interface which alternates the role of the MFC between a strain energy harvester and a strain sensor by toggling the NC switch based on the voltage level across the storage capacitor.

The WSS is composed of a microcontroller (MCU) and a buffer which are switched on and off simultaneously by the EAI. The MCU used in the WSS is Jennic JN5148, which has a $2.4 \mathrm{GHz}$ IEEE 802.15.4 compliant transceiver to communicate wirelessly using ZigBee protocol with transmission current of around $13 \mathrm{~mA}$ [18]. The transmit power was set to $2.5 \mathrm{dBm}$ to ensure successful transmission over a long distance. The MCU has four 12-bit analogue-todigital converter (ADC) pins with a reference voltage of $2.4 \mathrm{~V}$ to read analogue signals and a wide operating voltage range of 2.4 to $3.6 \mathrm{~V}$. Given that the maximum voltage that the PMM can provide is no more than $3.3 \mathrm{~V}$ and to provide some safety margin for not exceeding the maximum operating voltage of the WSS, the low and high threshold voltages of the EAI are chosen to be $2.5 \mathrm{~V}$ and $3.15 \mathrm{~V}$, respectively. Data received at the receiving hub is the voltage $V_{\mathrm{RD}}$ across the resistor $R_{\mathrm{D} 2}$ of the voltage divider, which represents the strain measurement in the implemented system.

Piezoelectric energy harvesters (PEHs) are usually characterized as high output voltage devices [12], [19]. When the NC switch is opened so that the MFC is used as a strain sensor, direct connection of the MFC to the ADC pin of MCU for strain measurement is likely to damage the MCU. Therefore, a resistive network formed by low noise and good temperature stability thin film resistors $R_{\mathrm{D} 1}$ and $R_{\mathrm{D} 2}$ is added in parallel with the PMM at the rectifier output. The resistors are used as a voltage divider where the voltage $V_{\mathrm{RD}}$ across $R_{\mathrm{D} 2}$ is a small fraction of the rectified voltage generated by the MFC, which is safe as an input to the MCU. There is an advantage of the voltage divider in that it simplifies the circuit implementation without requiring the WSS to withstand high peak-to-peak voltage and response to both positive and negative voltage generated by the MFC. In addition, $V_{\mathrm{RD}}$ is taken as the sensor reading by interfacing with a buffer (LPV521) to prevent loading effect on the MFC due to the low impedance of the ADC pin of 5 to $10 \mathrm{k} \Omega$. Resistive network with a large resistance is used to ensure that there is little influence of the resistive network on the voltage generated by the MFC and the MFC is in open-circuit when used as a strain 
sensor. The whole sensing chain which comprises the FB rectifier with low reverse current, low noise resistors, and a high open-loop gain buffer for high linearity [20] with input signals at low frequency range of less than a few kilohertz will have negligible error to the measurement results and this will be verified in Section IV.D.

\section{Analyses of MFC as Strain Sensor and Energy Harvester}

Fig. 3 shows the general illustration of using a single piece of MFC as a multifunctional device for strain sensing or energy harvesting under an in-plane strain. It is known that when the MFC experiences a strain in an open-circuit condition, as shown in Fig. 3(a), a voltage will be generated across the electrodes.

For a 31 operational mode of the MFC, the amplitude of the open-circuit voltage $V_{\mathrm{OC}}$ generated can be given by (1) [6]:

$$
\left|V_{\mathrm{OC}}\right|=\frac{Y h\left|d_{31}\right|}{e_{33}^{\sigma}}|\varepsilon|
$$

where $Y$ is the Young's modulus, $h$ is the thickness of the piezoelectric material, $d_{31}$ is the piezoelectric charge coefficient, $e_{33}^{\sigma}$ is the dielectric permittivity of the piezoelectric material under a constant stress $(\sigma)$ condition, and $\varepsilon$ is the strain experienced by the MFC.

In the implemented system, the open-circuit voltage $V_{\mathrm{OC}}$ is used to determine the experienced strain of the MFC as there is a one-to-one relationship between them without loading effect on the MFC.

When the MFC is connected to an external load as an energy harvester, as shown in Fig. 3(b), the power generated by the MFC will be transferred to the load $Z_{\mathrm{L}}$. If the MFC is left open-circuited $\left(Z_{\mathrm{L}}=\infty\right)$, the power that can be transferred will be zero. This indicates that there is no power generated by the MFC when it is used as a sensor.

It should be mentioned that the amplitude of the voltage generated by the MFC as an energy harvester is lower than its open-circuit voltage because the external load $Z_{\mathrm{L}}$ and intrinsic impedance $Z_{\mathrm{S}}$ of the MFC form a voltage divider.

\section{Measurement ANd VAlidation Methods}

\section{A. Experimental Setup}

The experimental setup is shown in Fig. 4. The MFC chosen for this study is a M8528-P2 MFC from Smart Material GmbH - Dresden, Germany. The MFC was bonded to the center of a carbon fiber composite substrate. The composite substrate was held at both ends by the grips of an Instron testing machine. The Instron testing machine was used to apply sinusoidal strain loadings with different peak strains of $150 \mu \varepsilon, 200 \mu \varepsilon$, and $250 \mu \varepsilon$ at 5 and $10 \mathrm{~Hz}$ onto the carbon fiber composite for characterization of the implemented system performance to different strain loadings and frequencies to simulate the possible strain loadings that will be experienced by engineering structures such as aircrafts [5] and bridges [21], which is typically less than $300 \mu \varepsilon$ with frequency of 1 to $10 \mathrm{~Hz}$. Strains experienced by the carbon fiber composite substrate were measured by using both the implemented system and a commercial extensometer which was attached to the side of the substrate for comparison and verification of the accuracy of the strain measurement obtained by the implemented system.

All the electrical voltage and current were measured using Keithley 2612B sourcemeter units (SMUs) which were connected to a computer. The computer was used to control the SMUs and record all the measurement data via an in-house developed LabVIEW interface. A receiving hub, not shown in Fig. 4, positioned about 4 meters away from the implemented system, which receives data transmitted wirelessly from the WSS, was connected with another computer to record the measured data transmitted by the WSS when the MFC is used as a strain sensor. The implemented system was built using commercially available discrete components on breadboards for ease of measurement.

\section{B. Validation Methods}

The voltage generated $v_{\mathrm{g}}$ by the MFC and the voltage $V_{\mathrm{CS}}$ across the storage capacitor were simultaneously measured to verify the time-multiplexing operation of the implemented system in using the MFC as a strain energy harvester and a strain sensor at the appropriate time slots associated with the threshold voltages. Based on Fig. 2 and the explanation in

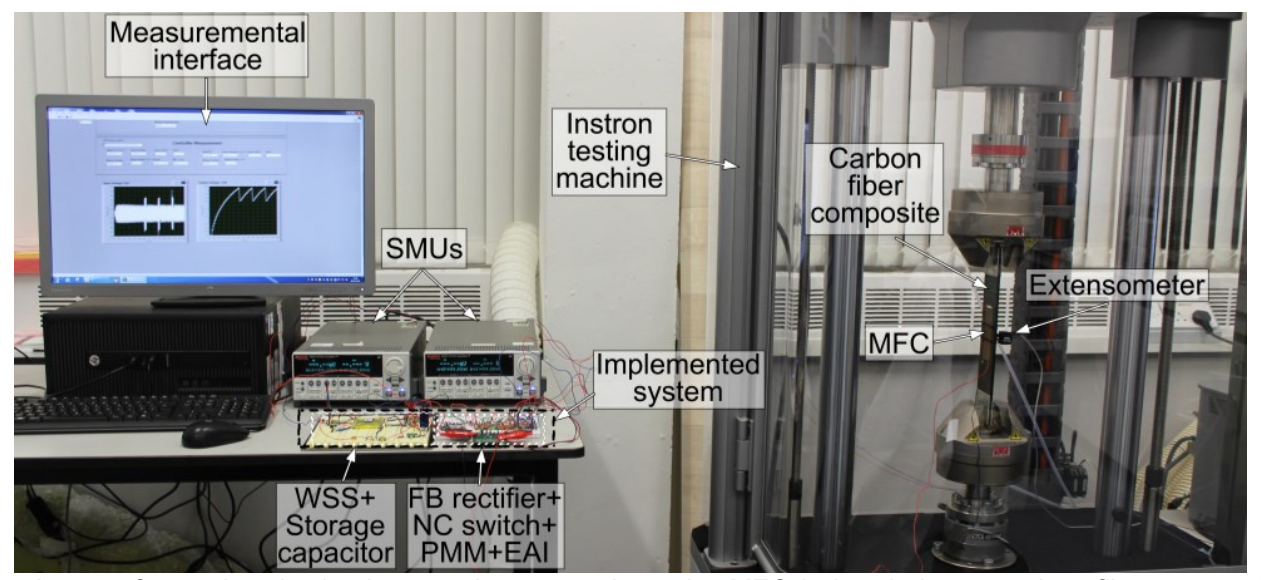

Fig. 4. The experimental setup for testing the implemented system where the MFC is bonded to a carbon fiber composite substrate which is mounted in the Instron machine which applies a strain loading onto the substrate. 
Section II.C, when the MFC is used as a strain sensor and the WSS is on, it is expected that: (1) the voltage $v_{\mathrm{g}}$ generated by the MFC is higher and have a shorter time slot than the voltage generated by the MFC when the MFC is used as a strain energy harvester and the WSS is off, and (2) the voltage $V_{\mathrm{CS}}$ decreases since energy is being drained from the storage capacitor and the MFC has been disconnected from the PMM. Furthermore, based on Fig. 2, when the MFC is being used as an energy harvester and the WSS is off, it is also expected that the voltage $V_{\mathrm{CS}}$ across the storage capacitor will increase since energy harvested by the PMM from the MFC, is stored into the storage capacitor.

\section{Power Generation of MFC used as Energy Harvester}

Energy generated by the MFC $E_{\mathrm{g}}$, energy output from the PMM $E_{\text {out-PMM, }}$ energy consumed by the WSS $E_{\mathrm{W}}$, and energy remained in the storage capacitor $E_{\mathrm{W}}$ were calculated as follows:

$$
\begin{gathered}
E_{\mathrm{g}}\left(t_{N}\right)=\sum_{k=1}^{N} v_{\mathrm{g}}\left(t_{k}\right) i_{\mathrm{g}}\left(t_{k}\right) \Delta t \\
E_{\text {out-PMM }}\left(t_{N}\right)=\sum_{k=1}^{N} v_{\mathrm{CS}}\left(t_{k}\right) i_{\text {out }}\left(t_{k}\right) \Delta t \\
E_{\mathrm{W}}\left(t_{N}\right)=\sum_{k=1}^{N} v_{\mathrm{W}}\left(t_{k}\right) i_{\mathrm{W}}\left(t_{k}\right) \Delta t \\
E_{\mathrm{C}}\left(t_{k}\right)=E_{\text {out-PMM }}\left(t_{k}\right)-E_{\mathrm{W}}\left(t_{k}\right)
\end{gathered}
$$

where $v_{\mathrm{g}}\left(t_{k}\right)$ and $i_{\mathrm{g}}\left(t_{k}\right)$ are the voltage and current generated by the MFC respectively, $v_{\mathrm{CS}}\left(t_{k}\right)$ is the voltage across the storage capacitor, $i_{\text {out }}\left(t_{\mathrm{k}}\right)$ is the output current from the PMM, $v_{\mathrm{W}}\left(t_{k}\right)$ is the voltage across the $V_{\mathrm{DD}}$ and GND pins of the MCU, $i_{\mathrm{W}}\left(t_{k}\right)$ is the current drawn by the WSS, and $\Delta t$ is the sampling period of the SMU, and is $1 \mathrm{~ms}$ for all the measured cases.

Average power generated by the MFC under the applied strain loading was calculated using (6):

$$
P_{\mathrm{g}}=\frac{E_{\mathrm{g}}\left(t_{N}\right)}{t_{N}}
$$

where $t_{\mathrm{N}}$ is the time duration of the measurement. Energy generated by the MFC and distributed in the implemented system, calculated using the measured voltages and currents, was also used to validate the time-multiplexing operation of the MFC as a multifunctional device. The increase, decrease or stall of the energy is related to the MFC functionality as an energy harvester or a sensor and whether the WSS is off or on.

The efficiency of the PMM was also calculated using (7):

TABLE I

PARAMETERS OF THE MFC AND CIRCUIT COMPONENTS

\begin{tabular}{cc}
\hline \hline Symbols & Parameters \\
\hline$Y$ & $30.34 \times 10^{9} \mathrm{~Pa}$ \\
$h$ & $300 \times 10^{-6} \mathrm{~m}$ \\
$d_{31}$ & $-1.7 \times 10^{-10} \mathrm{C} / \mathrm{N}$ \\
$e_{33}^{\sigma}$ & $1.37 \times 10^{-8} \mathrm{~F} / \mathrm{m}$ \\
$\mathrm{FVF}$ & 0.86 \\
$R_{\mathrm{D} 1}$ & $20 \mathrm{M} \Omega$ \\
$R_{\mathrm{D} 2}$ & $1.37 \mathrm{M} \Omega$ \\
$V_{\mathrm{F}}$ & $1.2 \mathrm{~V}$ \\
\hline \hline
\end{tabular}

$$
\eta=\frac{E_{\text {out-PMM }}\left(t_{N}\right)}{E_{\mathrm{g}}\left(t_{N}\right)} \times 100 \%
$$

\section{Voltage Generation of MFC as Strain Sensor}

The voltage generated by the MFC when used as a strain sensor in the implemented system was measured by the sourcemeter and compared with the voltage generated by the MFC in an open-circuit condition under the same strain loading condition to characterize the loading effect of the implemented circuit on the MFC.

The calculated amplitude of the open-circuit voltage, $V_{\text {open, }}$ generated by the MFC when used as a strain sensor, is calculated using the measured $V_{\mathrm{RD}}$ and based on (8):

$$
\left|V_{\text {open }}\right|=\left|V_{\mathrm{RD}}\left(\frac{R_{\mathrm{D} 1}+R_{\mathrm{D} 2}}{R_{\mathrm{D} 2}}\right)+V_{\mathrm{F}}\right|
$$

where $V_{\mathrm{F}}$ is the forward voltage drop across the FB rectifier.

\section{E. Calculation of Strain from the Measured Voltage}

In order to characterize the sensitivity of the dynamic strain sensing in the implemented system, that is, the relationship between the voltage $V_{\mathrm{RD}}$ and the strain, strain loading applied onto the composite substrate as obtained by the implemented system, was calculated in MATLAB based on (9), which is derived from (1) and (8):

$$
|\varepsilon|=\frac{e_{33}^{\sigma}}{Y h\left|d_{31}\right|}\left|V_{\mathrm{RD}}\left(\frac{R_{\mathrm{D} 1}+R_{\mathrm{D} 2}}{R_{\mathrm{D} 2}}\right)+V_{\mathrm{F}}\right| \mathrm{FVF}
$$

where FVF is the fiber volume fraction of the piezoelectric material in the MFC which contributes to the strain-voltage conversion [22]. Table I lists the parameters of the MFC and circuit component parameters used for the strain calculation.

\section{Results AND Discussions}

\section{A. Validation of the Time-multiplexing Operation}

Fig. 5 shows the measured voltage $v_{\mathrm{g}}$ generated by the MFC

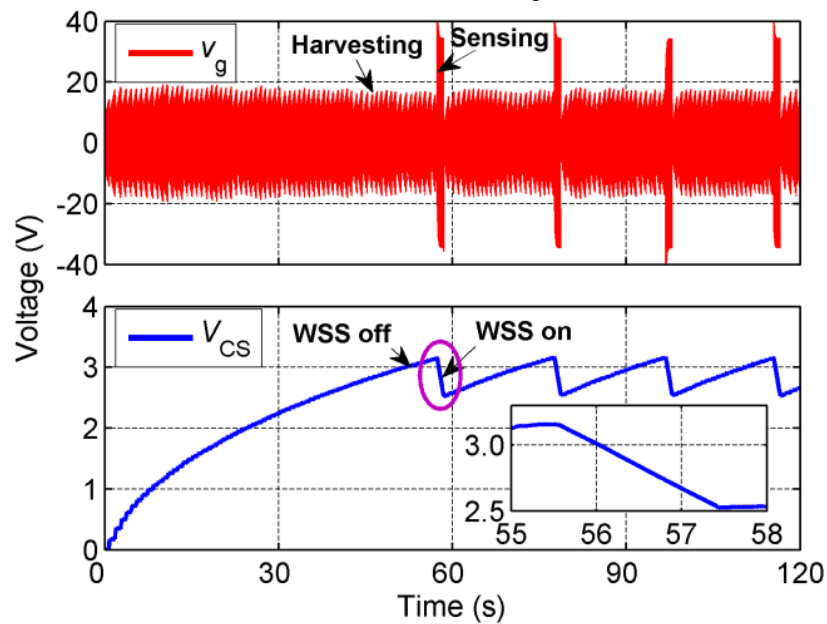

Fig. 5. Measured voltages $v_{g}$ generated by the MFC and $V_{\mathrm{Cs}}$ across the storage capacitor when the applied peak strain loading onto the composite substrate is $250 \mu \varepsilon$ at $10 \mathrm{~Hz}$. The MFC is for energy harvesting when the WSS is off, and for dynamic strain sensing when the WSS is on. The circled area is enlarged in the inset to show time duration of the WSS being kept on for dynamic strain sensing. 
and the measured voltage $V_{\mathrm{CS}}$ across the storage capacitor when the MFC is subjected to a peak strain loading of $250 \mu \varepsilon$ at $10 \mathrm{~Hz}$ as an example for the validation since tests with other loadings show the same trend. From Fig. 5, it can be seen that initially, the MFC was used as a strain energy harvester to charge up the storage capacitor since the voltage $V_{\mathrm{CS}}$ across the storage capacitor gradually increases from $0 \mathrm{~V}$ and the WSS is turned off, which allows energy to be accumulated. When $V_{\mathrm{CS}}$ reaches a high threshold voltage of $3.15 \mathrm{~V}$, the WSS becomes active to read the voltage generated by the MFC as strain measurement and starts to consume energy from the storage capacitor. During this period of time, the amplitude of $v_{\mathrm{g}}$ can be seen to increase significantly, indicating that the MFC is indeed disconnected from the PMM through the NC switch. Inset of Fig. 5 shows that the WSS was kept on for nearly 2 seconds for dynamic strain sensing before $V_{\mathrm{CS}}$ dropped to the low threshold voltage of $2.5 \mathrm{~V}$. Subsequently, the WSS is off and the MFC is used as a strain energy harvester again to recharge the storage capacitor where $V_{\mathrm{CS}}$ can be seen to gradually increase. The process of the MFC being used as a strain energy harvester to charge up the storage capacitor while the WSS is off and used as a strain sensor while the WSS is repeated as long as a strain loading is applied onto the MFC.

A repeated cycle sweep test using varying peak strain loadings of $150 \mu \varepsilon$ to $250 \mu \varepsilon$, and then back to $150 \mu \varepsilon$ at the frequency of $10 \mathrm{~Hz}$ was also carried out to determine the performance of the implemented system to varying strain levels which is most likely to happen in the real-world as shown in Fig. 6. The system is able to respond to the varying strain loadings as can be seen from $V_{\mathrm{CS}}$ which increases at a faster rate with a higher applied strain loading and vice versa. The implemented system is also able to measure the varying strain levels where the voltage generated by the MFC changes proportionally to the strain level, which agrees with (1) shown in the theoretical analyses in Section II.C.

Fig. 7 shows the energy distribution in the implemented system with the strain loading of $250 \mu \varepsilon$ at $10 \mathrm{~Hz}$ applied onto the carbon fiber composite substrate as an example as well. It can be seen that initially, the MFC was used as an energy harvester, and hence energy $E_{\mathrm{g}}$ generated by the MFC increases. Energy output from the PMM $E_{\text {out-PMM, }}$ and energy in the storage capacitor $E_{\mathrm{C}}$ increase as well since $E_{\mathrm{g}}$ generated by the MFC is harvested by the PMM and stored in the storage capacitor. The WSS consumes zero energy when it is off as seen from its energy consumption $E_{\mathrm{W}}$ which remains the same.

When sufficient energy has been accumulated to power the WSS, $E_{\mathrm{W}}$ can be seen to increase as the WSS is on and consumes energy from the storage capacitor, leading to a decrease in $E_{\mathrm{C}}$. In the designed circuit, the MFC is used as a sensor when the WSS is on. Therefore, there is no energy generated by the MFC in the studied circuit, leading to $E_{\mathrm{g}}$ to remain the same for the period of time when the WSS is on, as shown in the inset of Fig. 5. $E_{\mathrm{g}}, E_{\text {out-PMM, }}$ and $E_{\mathrm{C}}$ increase again when the MFC is used as an energy harvester and the WSS is off, with $E_{\mathrm{W}}$ unchanged as there is no energy consumed. $E_{\text {out-PMM }}$ shows similar trend as $E_{\mathrm{g}}$ but with lower

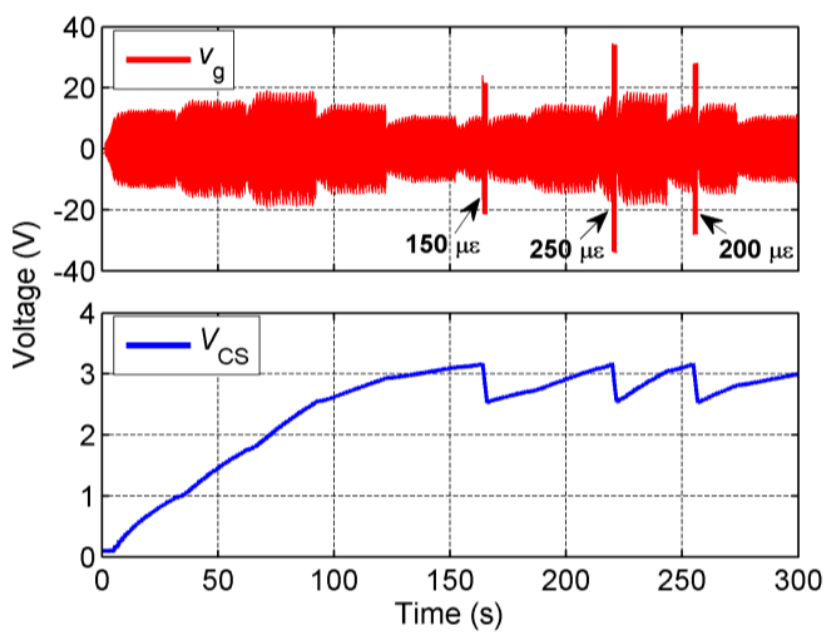

Fig. 6. Measured voltage generated by the MFC and the voltage $V_{C S}$ across the storage capacitor, when the MFC is subjected to varying peak strain levels in a sweep test.

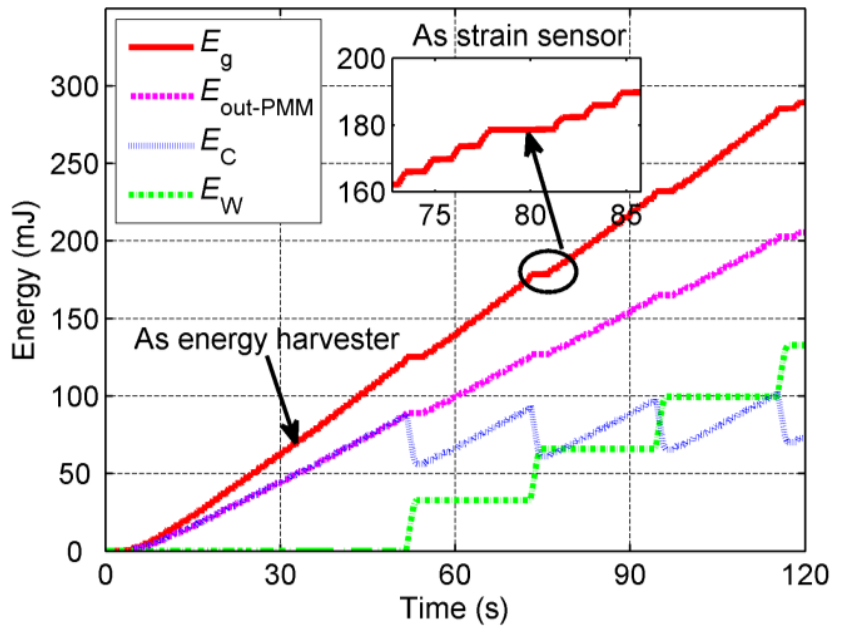

Fig. 7. Energy distributions in the implemented system with peak strain loading of $250 \mu \varepsilon$ at $10 \mathrm{~Hz}$ applied onto the composite substrate, where the circled area is enlarged and presented in the inset, showing there is no energy harvesting during the sensing period as the energy from the MFC remained unchanged.

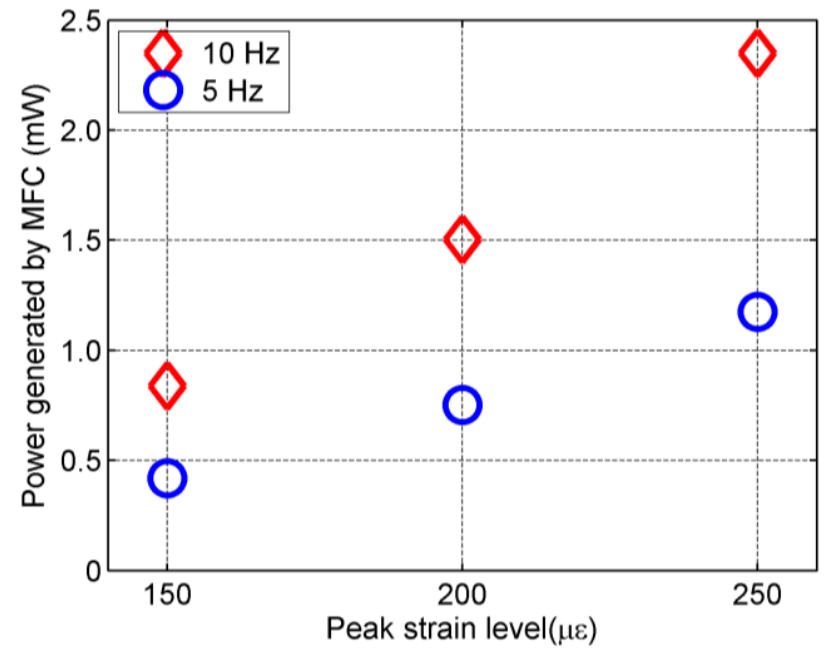

Fig. 8. Power generated by the MFC strain energy harvester when it is connected to the implemented system with different peak strain loadings of 150 to $250 \mu \varepsilon$ at $10 \mathrm{~Hz}$ (diamond) and $5 \mathrm{~Hz}$ (circle) applied onto the composite substrate. 
TABLE II

Performance of a Single MFC as Both the Energy HaRVester and StRain Sensor

\begin{tabular}{|c|c|c|c|c|c|}
\hline \multirow{3}{*}{$\begin{array}{c}\text { Peak strain } \\
\text { levels } \\
(\mu \varepsilon)\end{array}$} & \multicolumn{3}{|c|}{ Energy harvesting } & \multicolumn{2}{|l|}{ Strain sensing } \\
\hline & \multirow{2}{*}{$\begin{array}{l}\text { Energy per second per hertz } \\
(\mathrm{mJ} / \mathrm{s} / \mathrm{Hz})\end{array}$} & \multicolumn{2}{|c|}{ Efficiency of PMM (\%) } & \multirow{2}{*}{$\begin{array}{l}\text { Calculated peak voltages generated by MFC } \\
(\mathrm{V})\end{array}$} & \multirow{2}{*}{$\begin{array}{l}\text { Sensitivity } \\
(\mathrm{V} / \mu \varepsilon)\end{array}$} \\
\hline & & $5 \mathrm{~Hz}$ & $10 \mathrm{~Hz}$ & & \\
\hline 150 & 0.0903 & 74.47 & 76.83 & 19.73 & 0.1315 \\
\hline 200 & 0.1578 & 73.92 & 75.51 & 26.35 & 0.1317 \\
\hline 250 & 0.2411 & 72.29 & 74.48 & 32.79 & 0.1312 \\
\hline
\end{tabular}

amplitude because part of the energy from $E_{\mathrm{g}}$ is consumed by the PMM itself.

Since the leakage current is very low at a few microamperes, the effect of the leakage current of the storage capacitor is negligible compared with the current consumed by the WSS at a few milliamperes and is not considered here.

\section{B. Power Generated by MFC as Strain Energy Harvester}

Fig. 8 shows the power generated by the MFC when it is used as an energy harvester in the implemented system. The power generated was used to power up the WSS to take $V_{\mathrm{RD}}$ voltage reading as the strain measurement through $\mathrm{ADC}$ of the MCU and transmit it to the receiving hub. The power generated by the MFC is between 0.5 and $2.5 \mathrm{~mW}$ under the applied strain loadings at different frequencies. The power is also normalized to frequency to determine the power that can be generated by the MFC for a given strain level. The efficiencies of the PMM for the different applied strain levels and frequencies were calculated using (7). A summary of the energy harvesting performance of the implemented system under different testing conditions is given in Table II.

Results from Figs. 7 and 8 also show the rationale and viability of such a time-multiplexing operation to realize a multifunctional devices of both dynamic strain sensing and energy harvesting using just a piece of MFC. It can be seen that there is a mismatch between the power that the MFC can generate instantly and the energy demanded by the WSS. This means the MFC will not be able to instantly supply sufficient energy to the WSS on its own. Therefore, the WSS can only be powered on when sufficient amount of energy harvested by the MFC has been accumulated in the storage capacitor. Given

(a)

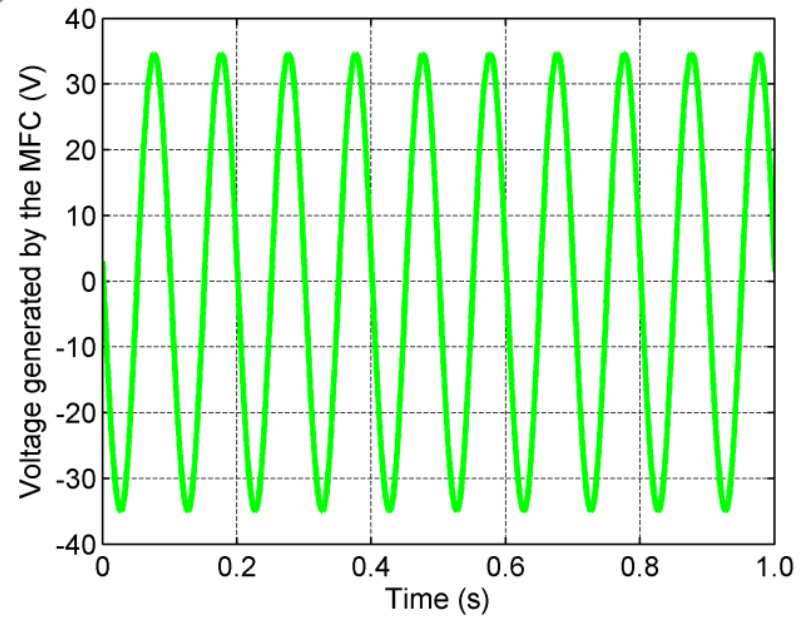

that the WSS is mainly acquiring the energy from the storage capacitor for its operation when it is on, the MFC is not required for energy harvesting and can be used for strain sensing. It should also be noted that in real-world applications, measurements can be taken in a duty-cycle mode with intervals ranging from a few minutes to a few hours [23], [24].

\section{Voltage Generation When MFC is used as Strain Sensor}

Fig. 9 shows the voltage generated by the MFC in the opencircuit condition and connected to the implemented system as a strain sensor, which is enlarged from Fig. 5, under the applied peak strain loading of $250 \mu \varepsilon$ at $10 \mathrm{~Hz}$ onto the carbon fiber composite substrate as an example. The waveforms of the voltage generated in both circuit configurations are almost identical up until around $57.4 \mathrm{~s}$ in Fig. 9 (b), indicating that the MFC is well isolated from the PMM and the resistors $R_{\mathrm{D} 1}$ and $R_{\mathrm{D} 2}$ have negligible loading effect on the MFC in the implemented circuit when the MFC is used as the strain sensor. Therefore, the voltage generated by the MFC as a strain sensor in the implemented system agrees well with the MFC in the open-circuit condition.

After around $57.4 \mathrm{~s}$ as shown in Fig. 9(b), there is a significant drop in the voltage generated by the MFC. This indicates that the $\mathrm{NC}$ switch is closed and the MFC is connected to the circuit as an energy harvester, as described in Section II.C. The drop in the voltage generated by the MFC is due to the MFC and the smoothing capacitor $C_{\mathrm{i}}$ are now in parallel, and thus the voltage of the MFC becomes the same as the low voltage at $C_{\mathrm{i}}$ initially. The voltage at $C_{\mathrm{i}}$ is low because no energy is transferred into $C_{\mathrm{i}}$ when the NC switch is opened for the MFC to function as the strain sensor and $C_{\mathrm{i}}$ is almost

(b)

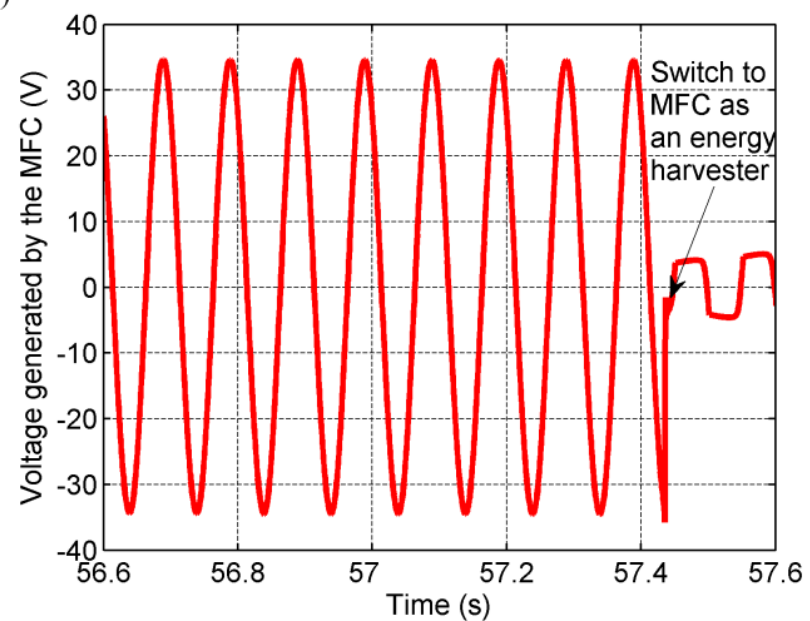

Fig. 9. Voltage generated by the MFC when it is (a) in an open-circuit and (b) used as a strain sensor in the implemented system (enlarged from Fig. 5) with a peak strain loading of $250 \mu \varepsilon$ at $10 \mathrm{~Hz}$ applied onto the composite substrate. 
fully discharged during that time. $C_{\mathrm{i}}$ will be gradually charged up and once it has been charged up to about half of the opencircuit voltage of the MFC, the energy transfer process begins with the voltage from the MFC being maintained at around half of the open-circuit voltage as shown in Fig. 5.

\section{Calculated Strain from the Measured Voltage}

To understand the accuracy, reproducibility, and symmetry

(a)

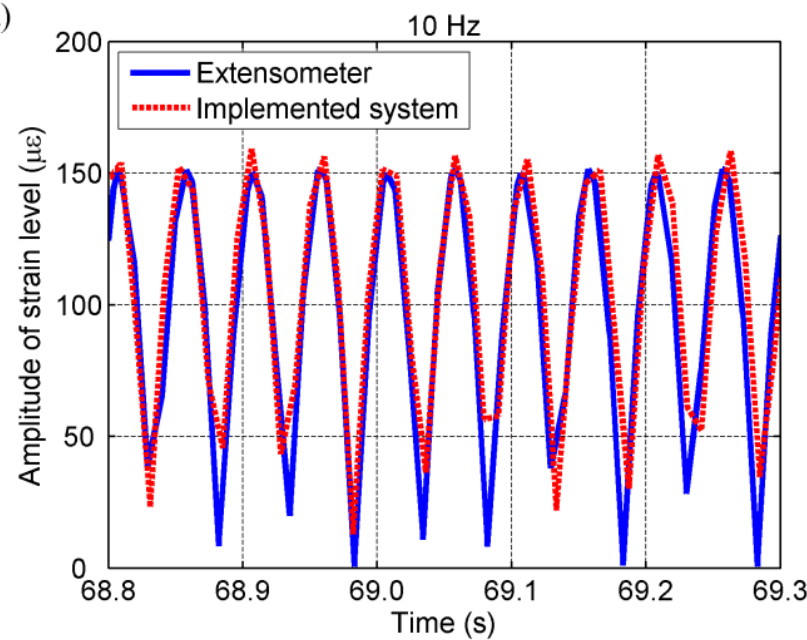

(b)

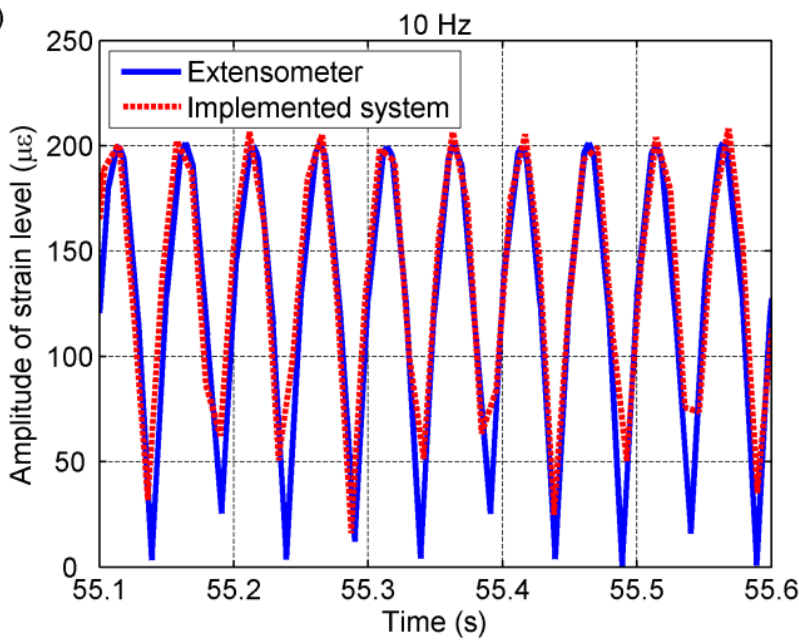

(c)

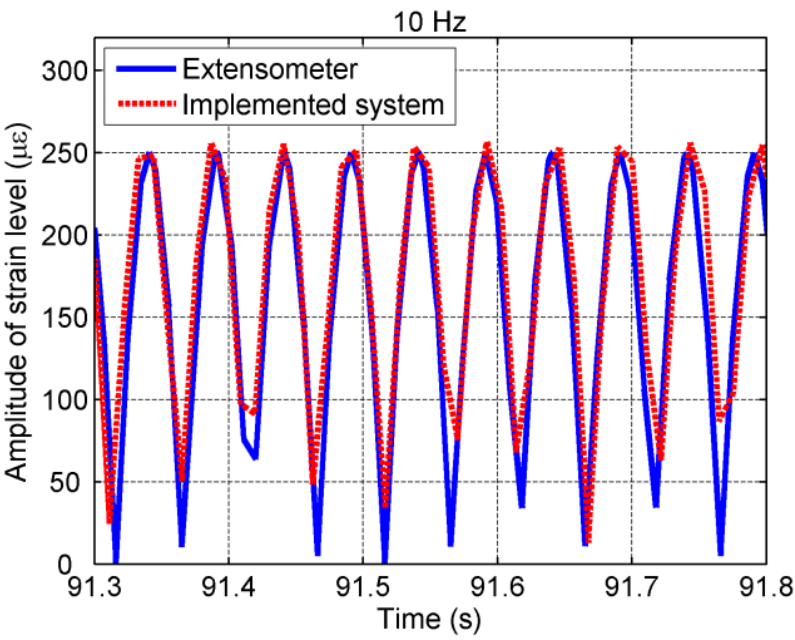

of the measurements made by the developed system, the measurements were made four times for each of the tested conditions and the obtained results were consistent, Fig. 5 showing one of the measured results. Fig. 10 shows the comparison between the calculated strains using (9) based on the measurements of $V_{\mathrm{RD}}$ by the implemented system and the measured strains using the commercial strain sensor of extensometer when the carbon fiber composite substrate was

(d)

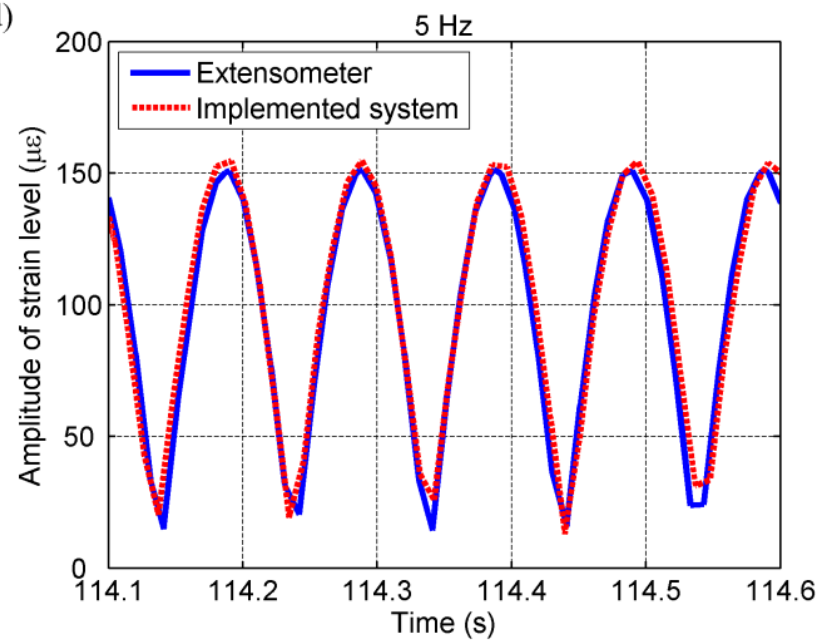

(e)

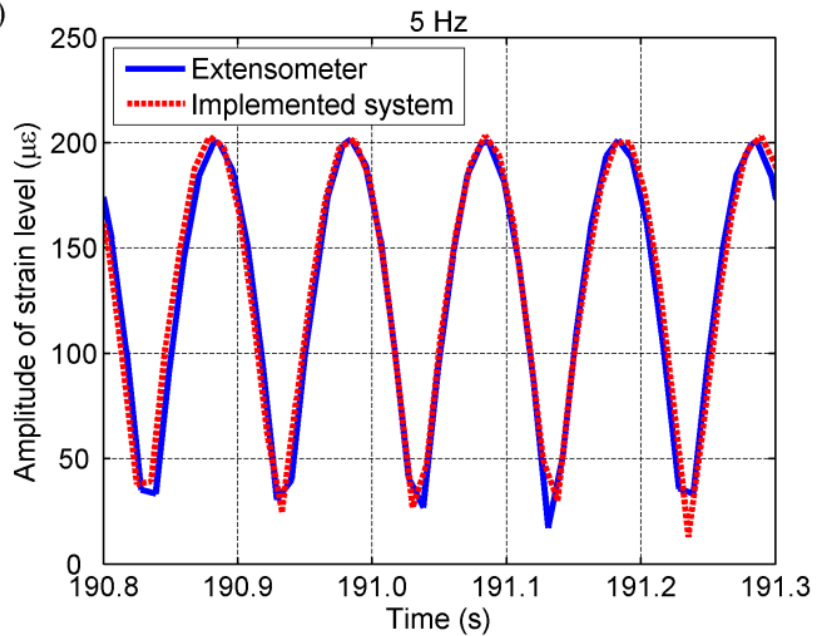

(f)

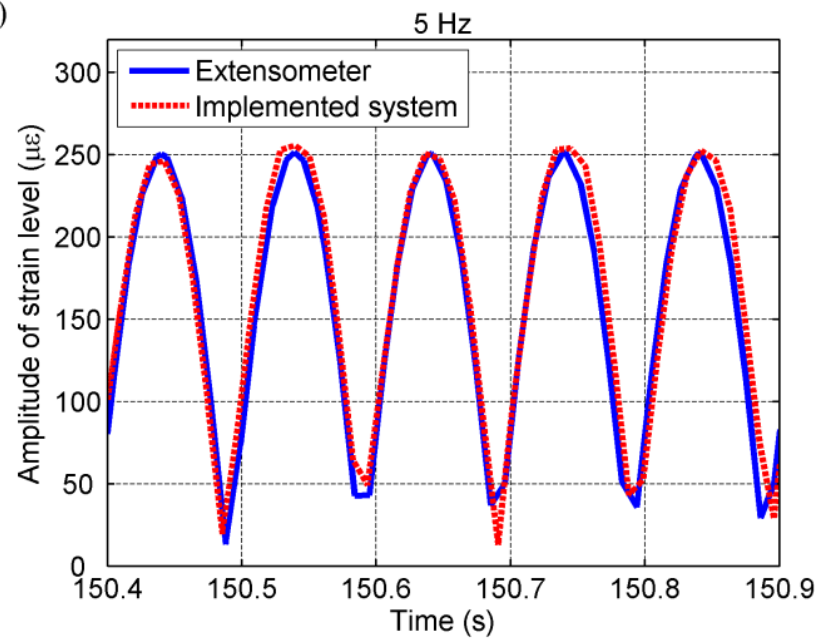

Fig. 10. Comparison of measured strains by the implemented system (dashed) and commercial strain sensor of extensometer (solid) when the carbon fiber composite substrate is subjected to peak strain loadings of $150 \mu \varepsilon, 200 \mu \varepsilon$, and $250 \mu \varepsilon$ at $10 \mathrm{~Hz}$ for (a), (b), and (c) respectively, as well as at $5 \mathrm{~Hz}$ for (d), (e), and (f) respectively. 
subjected to various peak strains loadings of $150 \mu \varepsilon, 200 \mu \varepsilon$, and $250 \mu \varepsilon$ at 10 and $5 \mathrm{~Hz}$, respectively. It can be observed that they match well with each other for all the tested conditions, showing good symmetry of the rectified waveform, accuracy, and repeatability of strain measurement using this developed method although there is a slightly discrepancy which is caused by the low sampling rate used. The low sampling rate is mainly limited by the commercial extensometer which has a maximum sampling rate of $100 \mathrm{~Hz}$. For fair comparison, the MCU in the WSS was set to have the same sampling rate at $100 \mathrm{~Hz}$ as well. The effect of the sampling rate can be verified by the comparison of the results in Fig. 10 with the same sampling rate but with different strain loading frequencies of 10 and $5 \mathrm{~Hz}$ where the strain waveforms at $5 \mathrm{~Hz}$ match better than those at $10 \mathrm{~Hz}$ due to the relatively higher sampling rate.

\section{E. System Performance}

All the measured peak strains by the extensometer and the implemented system in Fig. 10 were compared to determine the accuracy of the implemented system. The accuracy is around 97 to $99.99 \%$ for most of the time, with only a few points at 95.5 to $96.9 \%$, due to the low sampling rate and slightly different sampling time of both the implemented system and extensometer. Theoretical value of the voltage generated by the MFC for a given strain level using (1) is compared with the experimental voltage level inferred by the implemented system using (8) to determine the linearity of the implemented system. Fig. 11 shows that its output almost overlaps with the ideal curve, with a maximum difference of only $0.319 \%$. The reason that the implemented system can achieve high accuracy and linearity with no sign of fluctuations due to noise and component nonlinearities as shown in Fig. 10 and Fig. 11 is explained below.

The sensitivity of the MFC as a strain sensor was determined by dividing the amplitudes of the open-circuit voltage generated by the MFC at different strain levels calculated using (8) by the applied peak strain. The results are given in Table II, showing that the MFC as a strain sensor has a fairly high sensitivity of about $0.132 \mathrm{~V} / \mu \varepsilon$. The high output voltage from the MFC ensures that the signal-to-noise ratio is high without the need of a signal amplification circuit, which will amplify all the signals including noise. This simplifies the circuit design as can be seen from the implemented system in Fig. 1 where only a buffer and a MCU are used without the need to implement additional noise filtering circuit.

With fewer components introduced in the sensing chain, risks of measurement errors arising from noise such as thermal noise and current noise, usually rated at $\mathrm{nV} / \sqrt{\mathrm{Hz}}$ and $\mathrm{pA} /$ $\sqrt{\mathrm{Hz}}$, respectively [25] can be considered negligible since the noise floor is only a few nano to microvolts. The components used in the sensing chain are very stable against temperature variations as well. The material properties of the MFC are almost constant up to $60{ }^{\circ} \mathrm{C}$ [26]. The resistors have a temperature coefficient of 50 to $100 \mathrm{ppm} /{ }^{\circ} \mathrm{C}$ and the buffer used is also very stable with around $\pm 100 \mu \mathrm{V}$ offset when temperature changes from -40 to $85^{\circ} \mathrm{C}$. Therefore, the effects

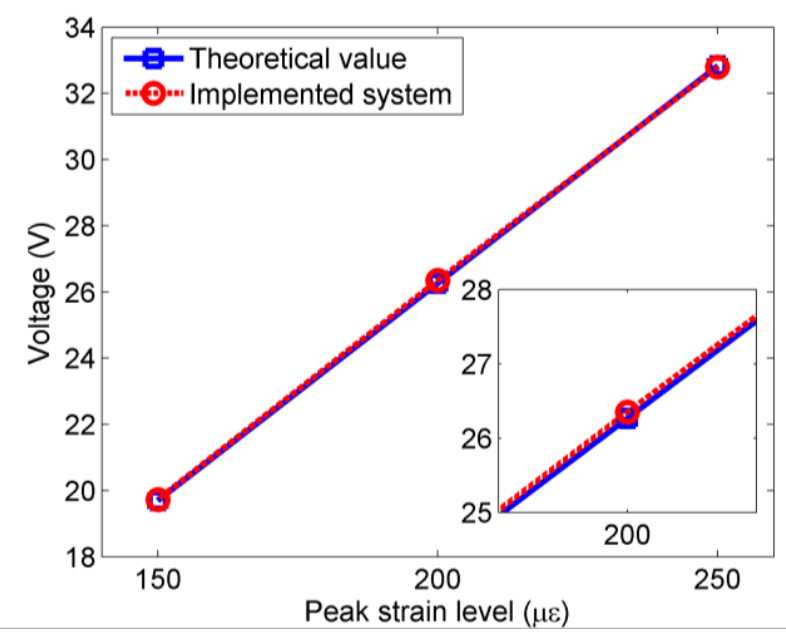

Fig. 11. Comparison of the output from the implemented system with a theoretical ideal value to determine the linearity of the sensing system. Inset shows the maximum difference between both values.

of temperature variations on these components are negligible in the implemented system for most of the time under normal operating conditions. The noise levels of around nano to microvolts and fluctuations due to temperature variations are also negligible for the 12-bit ADC which has a resolution of around $586 \mu \mathrm{V}$ or $0.00444 \mu \varepsilon$ per bit.

Although diodes have nonlinear characteristics where the forward voltage drop of the diodes used increases from around 0.5 to $0.7 \mathrm{~V}$ when the amplitude of current flowing through increases from 0.1 to $1 \mathrm{~mA}$ and decrease from around 0.6 to $0.4 \mathrm{~V}$ when temperature changes from -30 to $80{ }^{\circ} \mathrm{C}$, this can be translated to a maximum error of around $3 \mu \varepsilon$ or around 1.2 to $2 \%$ in the studied cases based on the obtained sensitivity of the MFC and calculated using (9). Given that the common range of strain measurements are a few hundred of microstrains and the strain limit of a structure is usually more than a thousand of microstrains [21], this error is negligible since the strain measurements made on a structure is to ensure that the structure maintains its structural integrity and maintenance can be carried out before the structure reaches its fatigue limit. Therefore, the nonlinearity of the diodes is negligible as well.

The $\mathrm{MFC}$ is required to generate a voltage which is sufficiently high to overcome the forward voltage drop $V_{\mathrm{F}}$ of the FB rectifier for the WSS to detect the output from the MFC. Therefore, the MFC has to experience a baseline strain level of around $15 \mu \varepsilon$ to generate a voltage signal which is detectable by the implemented system based on (1). If the system was to be powered up using energy harvesting, a minimum voltage of around $5 \mathrm{~V}$ is required, which in this case, is available when the peak strain level is around $37 \mu \varepsilon$. It is expected that when the temperature dependency of the measurement system was compensated and by using Schottky diodes with lower $V_{\mathrm{F}}$ as the FB rectifier, the baseline strain level experienced by the MFC which is detectable by the implemented system can be further improved to around $5 \mu \varepsilon$, and the whole system can be powered up at $27 \mu \varepsilon$. 


\section{CONCLUSIONS}

A single piece of MFC as a multifunctional device for both the strain energy harvesting and strain sensing in the context of energy-autonomous WSS has been presented in this paper. The multifunctional device is designed and implemented by using time-multiplexing operation where the MFC is used as a strain energy harvester and a strain sensor alternately at different time slots associated with different energy levels available to the system. The time-multiplexing operation is verified by experimental results. The accuracy of the strain measurements is also validated by the voltage generated by the MFC in open-circuit condition and comparison with a commercial extensometer as well since the strain waveforms are almost identical. The sensitivity of strain sensing is characterized and the MFC has a fairly high sensitivity of $0.132 \mathrm{~V} / \mu \varepsilon$. This allows fewer components being used especially for signal amplification to reduce the possible noise and nonlinearities introduced into the measured signal by the components along the sensing chain.

The system is easily implemented using the previously reported system architecture of energy harvesting powered sensing system but with an additional switch and a strain reading circuit in conjunction with a voltage supervisor to realize the time-multiplexing operation for dynamic strain sensing and energy harvesting at different time slots. Therefore, the proposed system offers the flexibility and ease to be integrated with existing systems, saving time and cost. The implemented system can be fully autonomous for dynamic strain measurement applications such as structural health monitoring of ground transportations, aircrafts, and civil infrastructures. The novel implementation of a single piece of MFC as sensor and energy harvester offers simplicity in multifunctional realization of autonomous sensing and energy harvesting in the context of energy harvesting powered dynamic strain sensing system, which is beneficial especially when wide deployment of the system within a big structure is required as a material-saving and cost effective solution especially in this Internet of Things (IoT) era.

\section{REFERENCES}

[1] J. P. Lynch, "An overview of wireless structural health monitoring for civil structures," Phil. Trans. R. Soc. A, vol. 365, no. 1851, pp. 345-372, 2007.

[2] C. Liu, J. Teng, and N. Wu, "A wireless strain sensor network for structural health monitoring," Shock Vib., vol. 2015, pp. 740471 (13 pp), 2015.

[3] G. Park, T. Rosing, M. D. Todd, C. R. Farrar, and W. Hodgkiss, "Energy harvesting for structural health monitoring sensor networks," $J$. Infrastruct. Syst., vol. 14, no. 1, pp. 64-79, 2008.

[4] J. Yao, Y. Y. M. Hew, A. Mears, and H. Huang, "Strain gauge-enable wireless vibration sensor remotely powered by light," IEEE Sensors J., vol. 15, no. 9, pp. 5185-5192, 2015.

[5] M. Pozzi, S. Guo, and M. Zhu, "Harvesting energy from the dynamic deformation of an aircraft wing under gust loading," in Proc. SPIE Health Monit. Struct. Biol. Syst., 2012, vol. 8348, pp. 834831-1-11.

[6] A. Giuliano, and M. Zhu, "A passive impedance matching interface using a PC permalloy coil for practically enhanced piezoelectric energy harvester performance at low frequency," IEEE Sensors J., vol. 14, no. 8, pp. 2773-2781, 2014.

[7] M. Shim, J. Kim, J. Jeong, S. Park, and C. Kim, "Self-powered $30 \mu \mathrm{W}$ to $10 \mathrm{~mW}$ piezoelectric energy harvesting system with $9.09 \mathrm{~ms} / \mathrm{V}$ maximum power point tracking time," IEEE J. Solid-State Circuits, vol. 50, no. 10, pp. 2367-2379, 2015.

[8] H. Gullapalli, V. S. M. Vemuru, A. Kumar, A. Botello-Mendez, R. Vajtai, M. Terrones, S. Nagarajaiah, and P. M. Ajayan, "Flexible piezoelectric $\mathrm{ZnO}$-paper nanocomposite strain sensor," Small, vol. 6, no. 15, pp. 1641-1646, 2010.

[9] J. R. Wait, and M. D. Todd, "Validation of macro fibre composites as strain sensors," in IMAC-XXV: Conf. \& Expo. Struct. Dyn., 2007, vol. 20533, pp. 1-12.

[10] C. A. Dan, P. Malinowski, P. Kudela, W. Ostachowicz, S. Opoka, M. Radzieński, and M. Mieloszyk, "Validation of macro fiber composites for strain measurements in structural health monitoring applications of complex aerospace structures," in Proc. 9th Int. Conf. Struct. Dyn., EYRODYN 2014, Porto, Portugal, 2014, pp. 2127-2134.

[11] D. Zhou, N. Kong, D. S. Ha, and D. J. Inman, "A self-powered wireless sensor node for structural health monitoring," in Proc. SPIE 7650, Health Monit. Struct. Biol. Syst., 2010, vol. 7650, pp. 765010 (12 pp).

[12] S. Park, C. Lee, H. Kim, H.-S. Yun, and S. Kwon, "Development of piezoelectric energy harvesting modules for impedance-based wireless structural health monitoring system," KSCE J. Civ. Eng., vol. 17, no. 4, pp. 746-752, 2013.

[13] Q. Tang, Q. He, M. Li, C. Dong, D. Xu, and X. Li, "Wireless alarm microsystem self-powered by vibration-threshold-triggered energy harvester," IEEE Trans. Ind. Electron., vol. 63, no. 4, pp. 2447-2456, 2016.

[14] V. Marsic, M. Zhu, and S. Williams, "Wireless sensor communication system with low power consumption for integration with energy harvesting technology," Telfor J., vol. 4, no. 2, pp. 89-94, 2012.

[15] Z. J. Chew, and M. Zhu, "Microwatt power consumption maximum power point tracking circuit using an analogue differentiator for piezoelectric energy harvesting," JPCS, vol. 660, no. 1, pp. 012022 (5 pp), 2015.

[16] F. Entezami, M. Zhu, and C. Politis, "How much energy needs for running energy harvesting powered wireless sensor node?," Energy Harvesting Syst., vol. 3, no. 3, pp. 197-203, 2016.

[17] Linear Technology, "LTC2935 - ultra-low power supervisor with power-fail output, selectable thresholds," Rep. no. LTC2935-1/2, Datasheet., 2008.

[18] T. Ruan, Z. J. Chew, and M. Zhu, "Energy-aware approaches for energy harvesting powered wireless sensor nodes," IEEE Sensors J., vol. 17, no. 7, pp. 2165-2173, 2017.

[19] G. K. Ottman, H. F. Hofmann, and G. A. Lesieutre, "Optimized piezoelectric energy harvesting circuit using step-down converter in discontinuous conduction mode," IEEE Trans. Power Electron., vol. 18, no. 2, pp. 696-703, 2003.

[20] M. Mousazadeh, K. Hadidi, and A. Khoei, "A highly linear CMOS buffer based on third harmonic cancellation," Analog Integr. Circuits Signal Process., vol. 86, no. 2, pp. 207-213, 2016.

[21] J. Xu, Y. Dong, Z. Zhang, S. Li, S. He, and H. Li, "Full scale strain monitoring of a suspension bridge using high performance distributed fiber optic sensors," Meas. Sci. Technol., vol. 27, no. 12, pp. 124017 ( 11 pp), 2016.

[22] M. A. Trindade, and A. Benjeddou, "Finite element characterisation of multilayer piezoelectric macro-fibre composites," Compos. Struct., vol. 151, pp. 47-57, 2016.

[23] N. De Battista, J. A. Rice, S.-H. Sim, J. M. W. Brownjohn, and P. H. Tan, "Embedded data processing in wireless sensor networks for structural health monitoring," Structural health monitoring 2013: A roadmap to intelligent structures, F.-K. Chang, Ed. DEStech Publications, 2013, pp. 1567-1574.

[24] S. Jang, H. Jo, S. Cho, K. Mechitov, J. A. Rice, S.-H. Sim, H.-J. Jung, C.-B. Yun, B. F. Spencer, Jr., and G. Agha, "Structural health monitoring of a cable-stayed bridge using smart sensor technology: Deployment and evaluation," Smart. Struct. Syst., vol. 6, no. 5-6, pp. 439-459, 2010.

[25] G. Erdi, "Amplifier techniques for combining low noise, precision, and high-speed performance," IEEE J. Solid-State Circuits, vol. 16, no. 6, pp. 653-661, 1981.

[26] R. B. Williams, D. J. Inman, and W. K. Wilkie, "Temperature-dependent thermoelastic properties for macro fiber composite actuators," J. Therm. Stresses, vol. 27, no. 10, pp. 903-915, 2004. 